\title{
The effect of exercise on cancer-related fatigue in cancer survivors: a systematic review and meta-analysis
}

This article was published in the following Dove Press journal:

Neuropsychiatric Disease and Treatment

\author{
Ellen Kessels ${ }^{1,2}$ \\ Olga Husson ${ }^{3}$ \\ Christina $M$ van der \\ Feltz-Cornelis ${ }^{1,2}$ \\ 'Tilburg University School of \\ Social Sciences, Tranzo Academic \\ Collaborative Centre "Geestdrift", \\ Tilburg University, ${ }^{2} \mathrm{Clinical}$ Centre \\ of Excellence for Body, Mind and \\ Health, GGz Breburg, Tilburg, \\ the Netherlands; ${ }^{3}$ The Institute of \\ Cancer Research, London, UK
}

Correspondence: Ellen Kessels GGz Breburg, Poolseweg 190, 4818 CG Breda, the Netherlands $\mathrm{Tel}+3 \mid 880161616$

Fax +3I88016I699

Email e.kessels@ggzbreburg.nl
Objective: The objective of the study was to conduct systematic review and meta-analysis to establish the effect of exercise interventions on cancer-related fatigue (CRF) in cancer survivors, compared to non-exercise intervention controls.

Methods: Trials published between January 1st 2000 and August 17th 2016 were included through PubMed database search and search of references. Eligible trials compared the effect of an exercise intervention on CRF compared to non-exercise intervention controls, with CRF as primary outcome and measured by validated self-report questionnaire, in cancer survivors not receiving palliative care. We evaluated risk of bias of individual trials following Cochrane Quality criteria. We performed a random-effects meta-analysis in the low risk of bias trials with intervention type, exercise intensity, adherence, and cancer type as moderators, and also performed meta-regression analyses and a sensitivity analysis including the high risk of bias trials.

Results: Out of 274 trials, 11 met the inclusion criteria, of which six had low risk of bias. Exercise improved CRF with large effect size (Cohen's $d 0.605,95 \%$ CI $0.235-0.975$ ) with no significant difference between types of cancer. Aerobic exercise ( $\Delta=1.009, \mathrm{CI} 0.222-1.797)$ showed a significantly greater effect than a combination of aerobic and resistance exercises $(\Delta=0.341$, CI $0.129-0.552)$. Moderator and meta-regression analyses showed high adherence yielding best improvements.

Conclusion: Exercise has a large effect on CRF in cancer survivors. Aerobic interventions with high adherence have the best result.

Keywords: exercise, cancer-related fatigue, cancer survivors, randomized clinical trials, systematic review, meta analysis

\section{Introduction}

\section{Background}

Cancer incidence is growing, with an incidence rate of 14.1 million in 2012 and 23.6 million new yearly cases predicted worldwide by $2030 .{ }^{1}$ Cancer incidence is highest in Denmark (338 cases per 100,000), followed by France (325/100,000), Australia $(323 / 100,000)$, the USA (318 cases per 100,000), and South Korea $(308 / 100,000)$ as top five countries. With growing incidence and current treatment possibilities, a growing number of people live beyond diagnosis. We define a person as a cancer survivor when he or she is living with and beyond a cancer diagnosis (like the US National Coalition for Cancer Survivorship). ${ }^{2}$

One of the major causes of distress in cancer survivors is cancer-related fatigue $(\mathrm{CRF}){ }^{3}$ Although in earlier research the prevalence of CRF as reported by patients in the USA ranged from $4 \%$ to $99 \%$, depending on the sample and assessment method, ${ }^{4-6}$ a recent USA-based study found a prevalence of $45 \%$ of moderate to severe CRF 
in cancer survivors. ${ }^{7}$ Several factors have been identified in research as contributing to fatigue, such as treatment, emotional distress, inactivity, and decondition. ${ }^{8}$ The nature of the fatigue is pervasive, as cancer survivors state "it's so much more than just feeling tired". 9 Several trials have shown that exercise has a positive effect on health-related quality of life, physiological and psychological side effects of treatment, ${ }^{10}$ and overall fitness, ${ }^{11}$ and may even increase survival rates. ${ }^{12}$ It has also been suggested that there are similarities ${ }^{13}$ among research on chronic fatigue syndrome (CFS), which shows that exercise can alleviate fatigue. ${ }^{14,15}$ It is estimated that up to $10 \%$ of the world population suffers from chronic fatigue. ${ }^{10}$ In all clinical settings including but not limited to oncology care, mental health care, general hospital care, and primary care, chronic fatigue prevalence rates of $40 \%-63 \%$ are reported. ${ }^{16}$ With so many people suffering from fatigue, it is important to be able to treat fatigue properly. A large number of studies have been conducted to test the effectiveness of treatments to reduce chronic fatigue, both in patients with and without chronic medical conditions, as it seems that there may be similarities between the chronic fatigue types.

A recent meta-analysis showed that both exercise and psychological treatment have small effect sizes in CRF, whereas medication has no effect. ${ }^{11}$ The effect depends on cancer treatment stage,${ }^{11}$ with most effect for exercise during cancer treatment, while psychotherapy has more effect after treatment.

Despite the evidence that exercise is beneficial, many cancer survivors do not engage in sufficient levels of exercise. Survivors report a significant decline in exercise after diagnosis, with less than half (48\%) engaging in a beneficial amount of exercise. ${ }^{17,18}$ Research on determinants of exercise levels is limited and shows contradictory results. For example, one study found being female and being older to be associated with decreased exercise ${ }^{19}$ another study found the opposite. ${ }^{20}$ Whether the nature of cancer treatment influences exercise levels of cancer survivors specifically is also up for debate. ${ }^{21}$ It might be that the exercise regimen is too strenuous; an ongoing debate concerns the question if patients should rest, or continue training despite malaise. It has been suggested in CFS patients that exercise improves fatigue but too much exercise has adverse effects, resulting in low adherence, ${ }^{22}$ post-exercise malaise is a frequent reason to stop an exercise regimen. ${ }^{23}$ Being fatigued is a valid reason for a person without cancer not to exercise, and the larger burden of CRF might make it even harder to exercise.

\section{Rationale}

$\mathrm{CRF}$, because of its persistence and interference with many aspects of daily life, ${ }^{24}$ even in cancer survivors with no evidence of active disease, ${ }^{25,26}$ leads to loss of work, limited social functioning including parenting, and even lower treatment adherence. In view of its high prevalence of $45 \%$, combined with growing survival rates, CRF can be considered as a major public health concern. An often dispensed advice is to exercise, but it is not clear how and with which intensity. Research is warranted that aims to clarify this.

A Cochrane systematic review and meta analysis by Cramp and Byron-Danie ${ }^{27}$ examined the effect of exercise on $\mathrm{CRF}$ in cancer survivors. Patients received non-palliative treatment or palliative treatment. Also, this review included CRF as secondary outcome. As we intend to establish the effect of exercise on CRF as a primary outcome in a population not suffering from specific end-of-life distress, we have only included trials evaluating exercise interventions versus non-exercise intervention controls, with CRF as a primary outcome measure. Hence, the results of this meta-analysis may provide the clinician with clear-cut information if exercise should be recommended, and what kind of exercise to recommend to cancer survivors not receiving palliative care.

\section{Objectives}

This study aims to provide a systematic review of RCTs evaluating the effect on CRF of exercise interventions versus non-exercise intervention controls in cancer survivors not receiving palliative care in trials evaluating $\mathrm{CRF}$ as a primary outcome measure. In our review, both level of exercise intensity and adherence are taken into account. The ensuing meta-analysis will provide a pooled estimate of the effect.

\section{Methods}

\section{Protocol and registration}

The review protocol has been registered in the Prospero Centre for reviews and dissemination ${ }^{28}$ under ID CRD42013003670. The systematic review and meta-analysis are performed and presented according to PRISMA Guidelines and with a PRISMA checklist (Figure S1). ${ }^{29}$

\section{Eligibility criteria}

An overview of participants, interventions, comparisons, outcomes, and study design (PICOS) is shown in Table 1. Patients were adults (18 years or older), regardless of sex, living with and beyond any cancer diagnosis. Patients were not receiving palliative care in the sense of symptom reduction in advanced disease, but may receive active treatment such as surgery, chemotherapy, or radiation therapy. Patients with metastatic disease were not included. Any length of follow-up was acceptable for inclusion and included in the data synthesis; however, for comparability of estimate of the 
Table I PICOS eligibility criteria

\begin{tabular}{|c|c|c|}
\hline Parameter & Inclusion criteria & Exclusion criteria \\
\hline \multirow[t]{3}{*}{ Patients } & Adults $>18$ years living with, through, or & Patients under 18 years of age \\
\hline & beyond a cancer diagnosis not receiving & Patients receiving palliative care \\
\hline & palliative care & Patients with metastatic cancer \\
\hline \multirow[t]{4}{*}{ Intervention } & Physical activity intervention of sufficient & An information letter/session \\
\hline & intensity as measured by METs & Education \\
\hline & & Yoga (of insufficient METs) \\
\hline & & Stretching \\
\hline \multirow[t]{2}{*}{ Comparator } & Non-exercise control group & Non-exercise control group receiving \\
\hline & & additional care, such as CBT \\
\hline \multirow[t]{3}{*}{ Outcomes } & Primary outcome: intensity of CRF as measured & CRF is not the primary outcome \\
\hline & by self-report questionnaire & \\
\hline & Secondary outcome: adherence & \\
\hline \multirow[t]{6}{*}{ Study design } & Randomized controlled trials published between & Non-randomized controlled trials \\
\hline & January Ist 2000 and August 17th 2016 & Retrospective, prospective, or \\
\hline & No language restrictions & concurrent cohort studies \\
\hline & & Cross-sectional studies \\
\hline & & Case reports \\
\hline & & Editorials and opinion pieces \\
\hline
\end{tabular}

Abbreviations: CBT, cognitive behavioral therapy; CRF, cancer-related fatigue; METs, metabolic equivalents of task; PICOS, patients, intervention, comparator, outcomes, study design.

effect, in the meta-analysis similar length of follow-up was chosen between studies.

\section{Information sources}

A PubMed database and a Cochrane Database search were performed. We also hand-searched the references noted by Cramp and Byron-Danie ${ }^{27}$ in their Cochrane review and each included article. Furthermore, we contacted study authors to identify relevant data for the analysis.

\section{Search}

The MeSH terms and free text terms used were "cancer" AND "fatigue" AND ("physical activity" OR "exercise") AND "randomized controlled trial". The search string was "cancer"[All Fields] AND "fatigue"[All Fields] AND ("physical activity"[All Fields] OR "exercise"[All Fields]) AND "randomized controlled trial”[All Fields]. For the Cochrane Database, the search was adapted to systematic reviews.

The search was limited to articles published between January 1st 2000 and August 17th 2016. There were no language or other search limitations.

\section{Study selection}

We included RCTs that evaluated any exercise intervention in any setting, individually or in a group, to study the effect on CRF in cancer survivors. The intervention was of sufficient intensity as measured in metabolic equivalent of the task (MET), thus not including stretching exercises. We also excluded yoga; although the majority of yoga sessions are of very light intensity, ${ }^{30}$ quite strenuous yoga exercise also exists and specific characteristics of the yoga intervention are usually not given. Comparisons were with a control group, not receiving any (major) exercise intervention or other intervention (eg, cognitive behavioral therapy). Primary outcome had to be CRF and was expressed in quantitative measures by a validated self-report questionnaire.

Titles and subsequent abstracts of trials were retrieved and screened in duplicate by two independent reviewers (EK and $\mathrm{OH}$ ) to identify trials that met the inclusion criteria. In case of disagreement, an independent third reviewer (CFC) gave her opinion regarding eligibility and the article was selected based on combination of the three. Subsequently, the full text of potentially eligible trials was retrieved and independently assessed for eligibility in duplicate by two independent reviewers $(\mathrm{EK}, \mathrm{OH})$ and the third reviewer in case of disagreement. Thereafter, risk of bias was assessed. For the meta-analysis, only RCTs with low risk of bias assessment were included. A flow chart will be presented in the subsection "Study selection".

\section{Data collection process}

Data was extracted by two authors (EK, $\mathrm{OH})$. When insufficient data were available in the full text, authors were contacted by email for further information.

\section{Data items}

The main variable for which data was sought is:

1) CRF severity, measured by self-report questionnaire validated to assess fatigue in cancer patients. 
Other moderating variables for which data were sought are:

1) Number of patients in intervention and control groups.

2) Type of exercise intervention.

3) Type of cancer.

4) Current stage of treatment and type of treatment received.

5) Intensity of exercise intervention in METs/hour; if MET was not mentioned, it was calculated $(\mathrm{OH})$ based on the described characteristics of the intervention. ${ }^{31}$

6) Adherence percentages; we made an assessment based on three aspects: level of reporting, adherence rates as a percentage based on the information in the trials regarding adherence, in terms of sessions attended, and if reporting was done by supervisor or self-report. Adherence level then was determined on a combined assessment of these three aspects, and split into three groups: low $(<60 \%)$, moderate $(60 \%-80 \%)$, and high $(>80 \%)$. This is indicated in a data extraction table (Table 2).

\section{Risk of bias in individual trials}

Two independent assessors (EK, OH) assessed the risk of bias of included trials on study level (not on outcome level) on the basis of the Cochrane Quality criteria, of which randomization is considered the most important. ${ }^{32}$ Any disagreement on eligibility was resolved through discussion with the third reviewer (CFC). Results are shown in a risk-of-bias table (Table 3 ). Trials with low risk of bias, adequately addressing more than half $(\geq 4 / 7)$ of the items, were included in the meta-analysis.

\section{Summary measures}

The principal summary measure was expressed as standardized difference in means (Cohen $d$ ) of CRF. These effect sizes indicate by how many standard units the intervention group is better off than the control group. The effect size $d$ is calculated by subtracting the average score of the control group $\left(M_{c}\right)$ from the average score of the experimental group $\left(\mathrm{M}_{\mathrm{e}}\right)$ and dividing the raw difference score by the pooled standard deviation of the experimental and control group. An effect size of 0.5 indicates that the mean of the experimental group is half a standard unit larger than the mean of the control group. It is generally assumed that an effect size of $0.56-1.2$ represents a large clinical effect, $0.33-0.55$ is medium, and below 0.33 is small. ${ }^{33}$ If trials reported more than one (validated) self-report measure for fatigue, we used only one in the analysis that was preferably also used in other trials. For the benefit of clinical relevance, we chose to use the outcomes at post-intervention, not at longer follow-up, in the meta-analysis.

\section{Synthesis of results}

We have provided a synthesis of results (Table 2) with an overview of type of cancer, intervention, and outcome. Subsequently, a random-effects meta-analysis was performed. Between-study heterogeneity was assessed by the observed dispersion, reflected by the $Q$-statistic. The $I^{2}$ statistic shows the percentage of total variation across trials that is the result of heterogeneity rather than chance and was used to quantify this dispersion. ${ }^{34}$ The statistical program Comprehensive Meta-Analysis v. $2^{35}$ was used for all analyses.

\section{Risk of bias across trials}

A test of publication bias was performed in order to assess evidence for publication bias and if the reported effect was valid. Publication bias was examined by constructing a Begg funnel plot ${ }^{36}$ and performing as the fail-safe $N .^{37}$

\section{Additional pre-envisioned moderator analyses}

We planned several moderator analyses, which are as follows:

\section{Type of intervention}

Type of intervention was split into three groups: aerobic, resistance, and a combination of resistance and aerobic exercise. Aerobic exercise is physical exercise that depends primarily on the aerobic energy-generating process. ${ }^{38}$ This refers to the use of oxygen to adequately meet energy demands during exercise via aerobic metabolism. ${ }^{39}$ Examples are walking, running, swimming, and cycling.

Resistance exercise is physical exercise that induces muscular contraction that builds the strength, endurance, and size of skeletal muscles as anaerobic activity associated with lactate production. Training commonly uses the technique of progressively increasing the force output of the muscle by increasing weight and using a variety of exercises to target specific muscle groups. ${ }^{40}$ Examples are weightlifting and rowing.

\section{Type of cancer}

Type of cancer was split into four groups: breast cancer, prostate cancer, gynecological cancer, and mixed.

\section{Intensity of exercise}

Intensity of exercise was split into high or low intensity (in METs/hour): ${ }^{32}$ high MET was operationalized as at least 


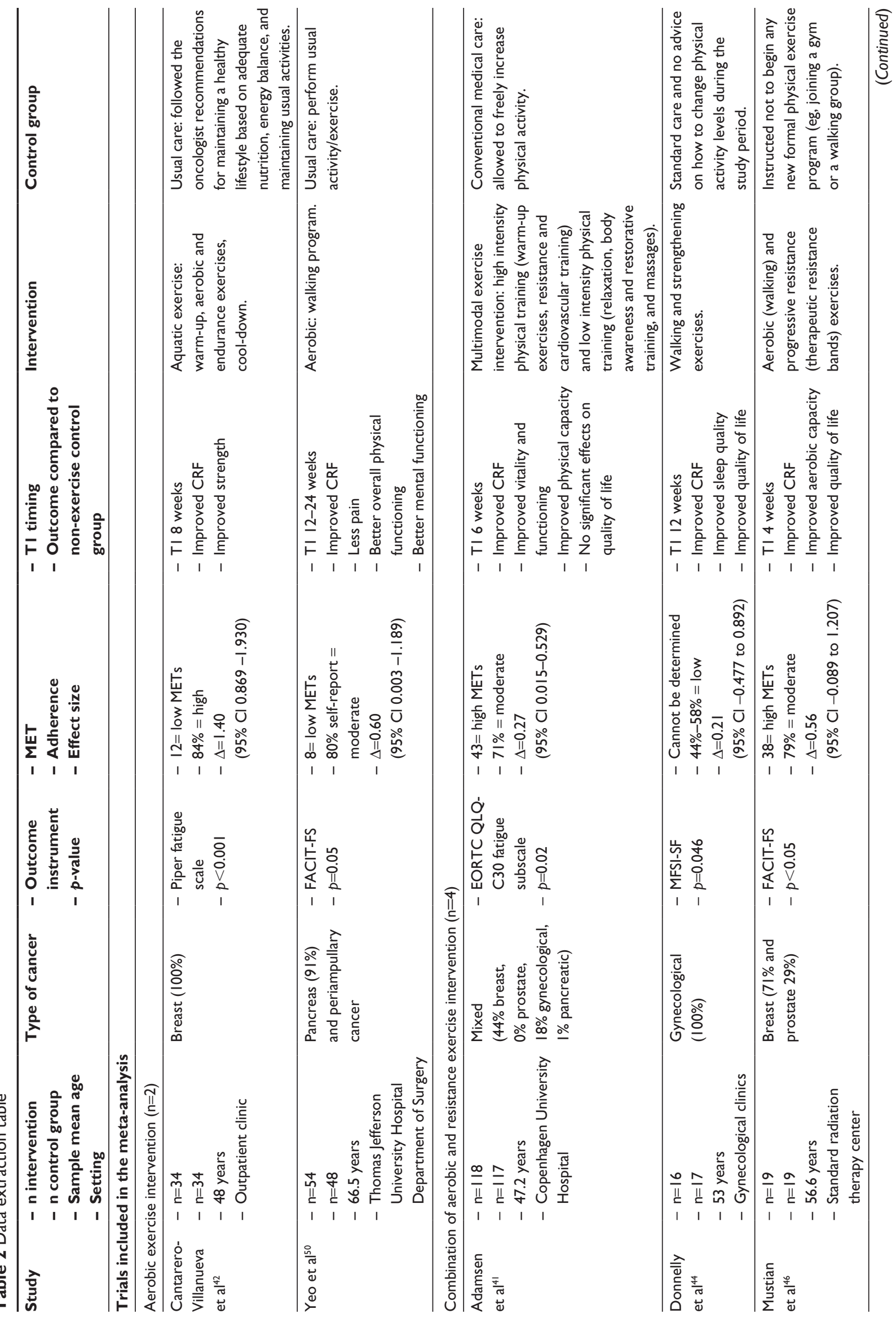




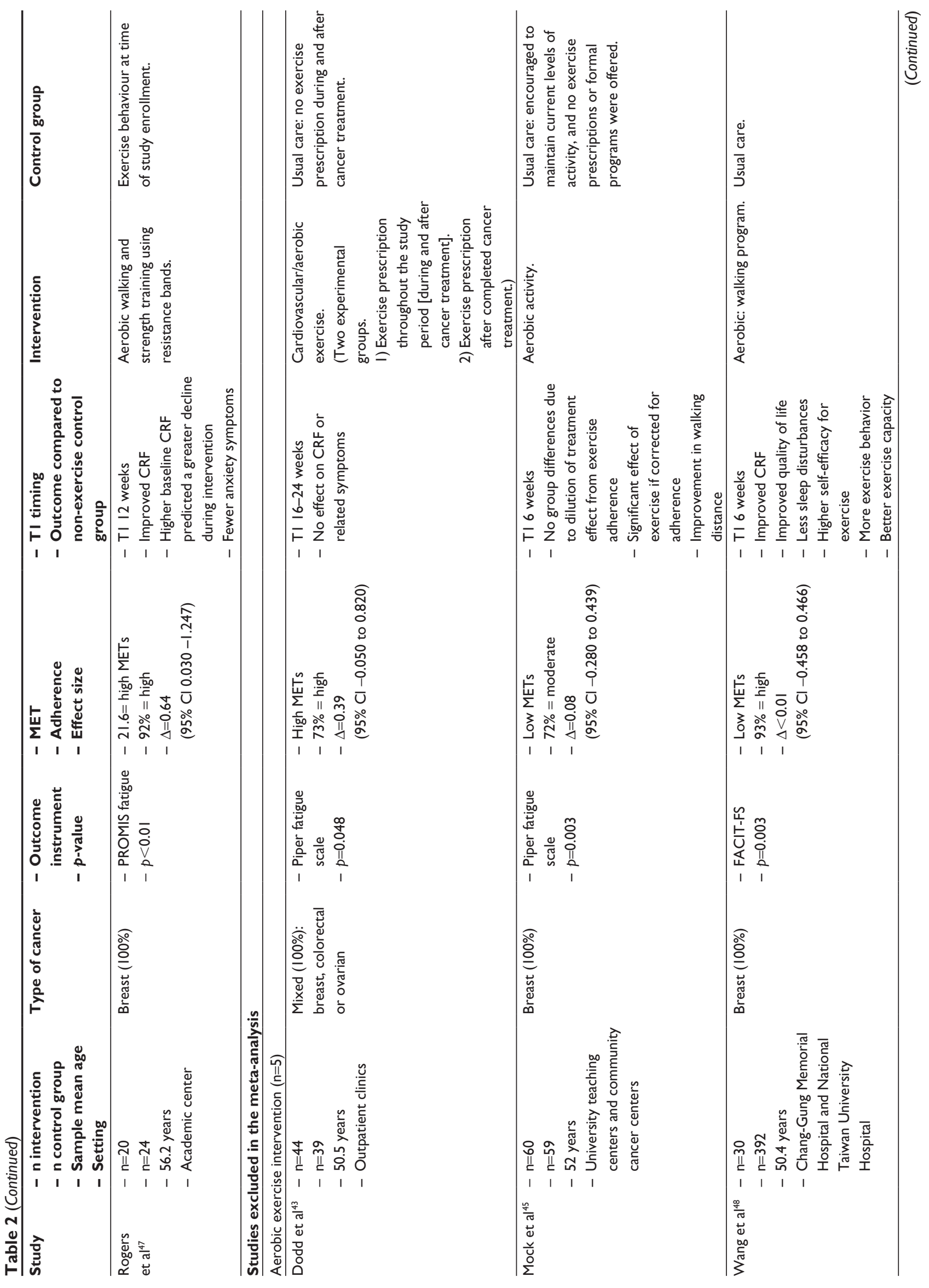




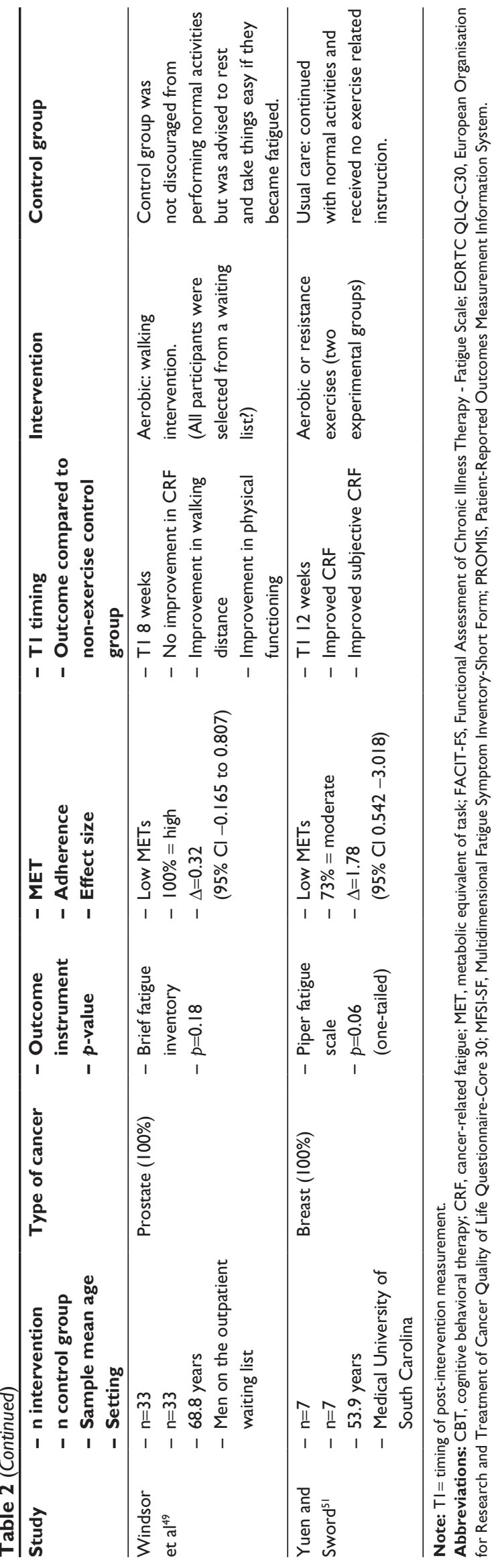

3.5 METs per day at least 5 days a week, adding up to $\geq 17.5$. Low MET was $<17.5$. For linear meta-regression, the actual MET scores were used.

\section{Adherence}

Adherence was split into three groups: low $(<60 \%)$, moderate $(60 \%-80 \%)$, and high $(>80 \%)$. For linear meta-regression, adherence percentages as reported in the trials were used.

After performing each analysis we examined heterogeneity. We performed a sensitivity analysis also including the articles with high risk of bias.

\section{Results Study selection}

The search strategy yielded 274 hits, 246 after checking for duplicates. Searching the Cochrane Database provided one systematic review, ${ }^{28}$ of which we hand-searched the references. After independently screening title and abstracts, 35 trials were included for independent full-text screening. We were not able to procure a translated version in English or Dutch for one study published in Korean. Twenty-four trials were excluded and finally 11 trials met the inclusion criteria as stated in the subsection "Study selection" and were included in the systematic review. Five of these were excluded because of high risk of bias and six were included in the meta-analysis. However, all 11 trials were included in the sensitivity analysis. See the PRISMA Flow Diagram (Figure 1) for an overview and exclusion reasons.

\section{Study characteristics}

All 11 trials were RCTs. ${ }^{41-52}$ In total, 788 patients were included in the trials, 411 of which received an exercise intervention and 377 were randomized in a non-exercise intervention control group as described in the section "Eligibility criteria". In five trials, ${ }^{42,44,46,47,49}$ patients were still undergoing cancer treatment during the intervention, in one study there was no active cancer treatment, ${ }^{43}$ and in the other five trials ${ }^{45,48,50-52}$ some patients were still undergoing cancer treatment but outcome reporting of fatigue scores was not differentiated between patients still undergoing cancer treatment or not. Post-intervention follow-up assessments of CRF varied from 4 weeks ${ }^{34}$ to $12-24$ weeks. ${ }^{50,51}$

\section{Risk of bias}

Risk of bias was determined for 11 trials on study level and is presented in Table 3. Several trials did not adequately address allocation concealment, missing data, and blinding. 


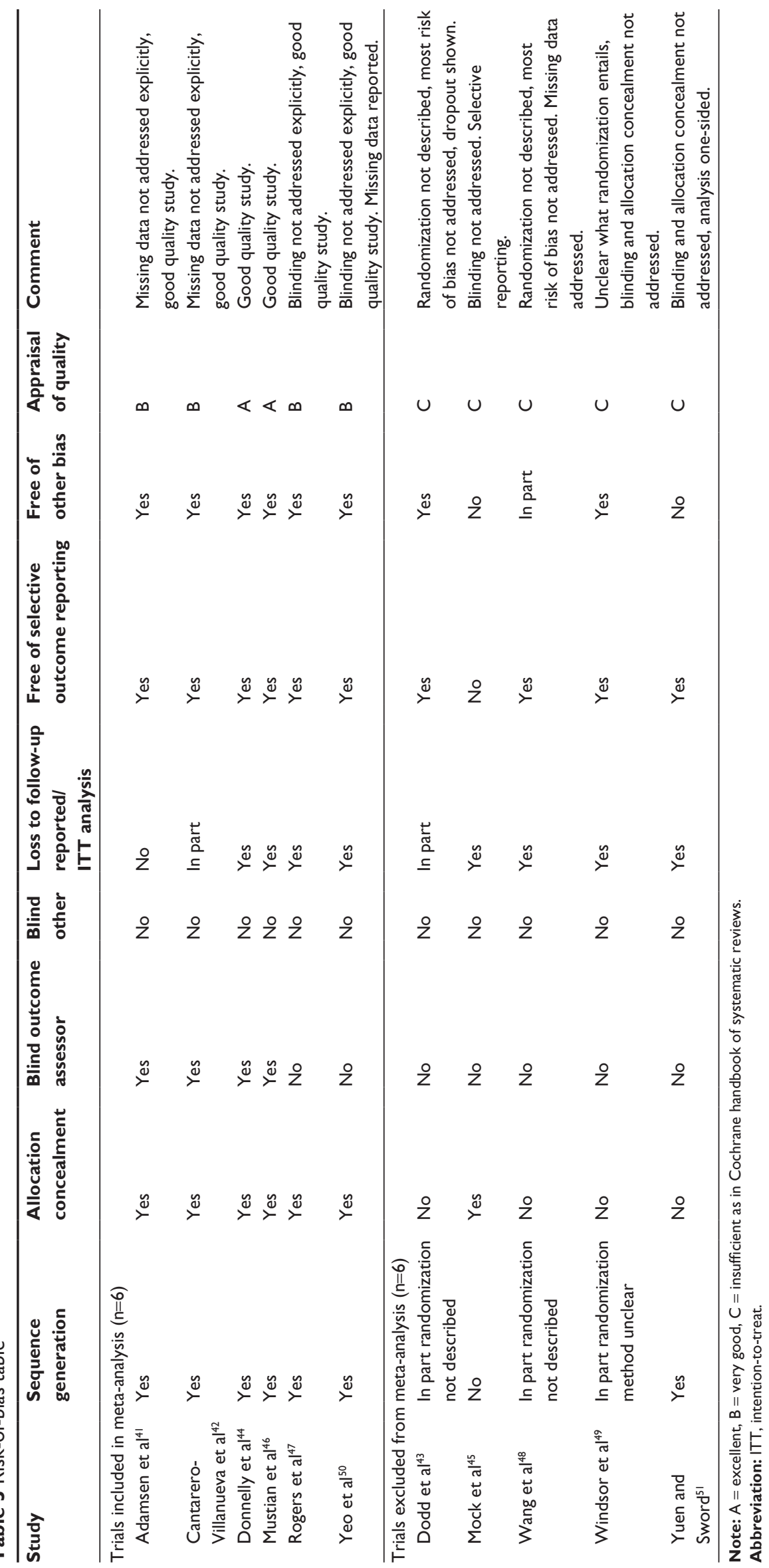


Trials not scoring the maximum may be underestimated due to lack of reporting. Six trials ${ }^{42,43,45,47,48,51}$ fulfilled the majority of criteria to eliminate risk of bias and were thus considered of sound quality. Five trials were assessed as being high risk of bias and were excluded from the initial meta-analysis. ${ }^{44,46,49,50,52}$ The risk of bias assessment showed that these trials failed to provide important information, such as not adequately describing the randomization procedure, ${ }^{49}$ testing one-sided while the research question was clearly two-sided, ${ }^{52}$ or otherwise as indicated.

\section{Results of individual trials}

The meta-analysis was performed first with the six low risk of bias trials. Later, a sensitivity analysis was performed with all 11 RCTs. The results are summarized in Table 2.

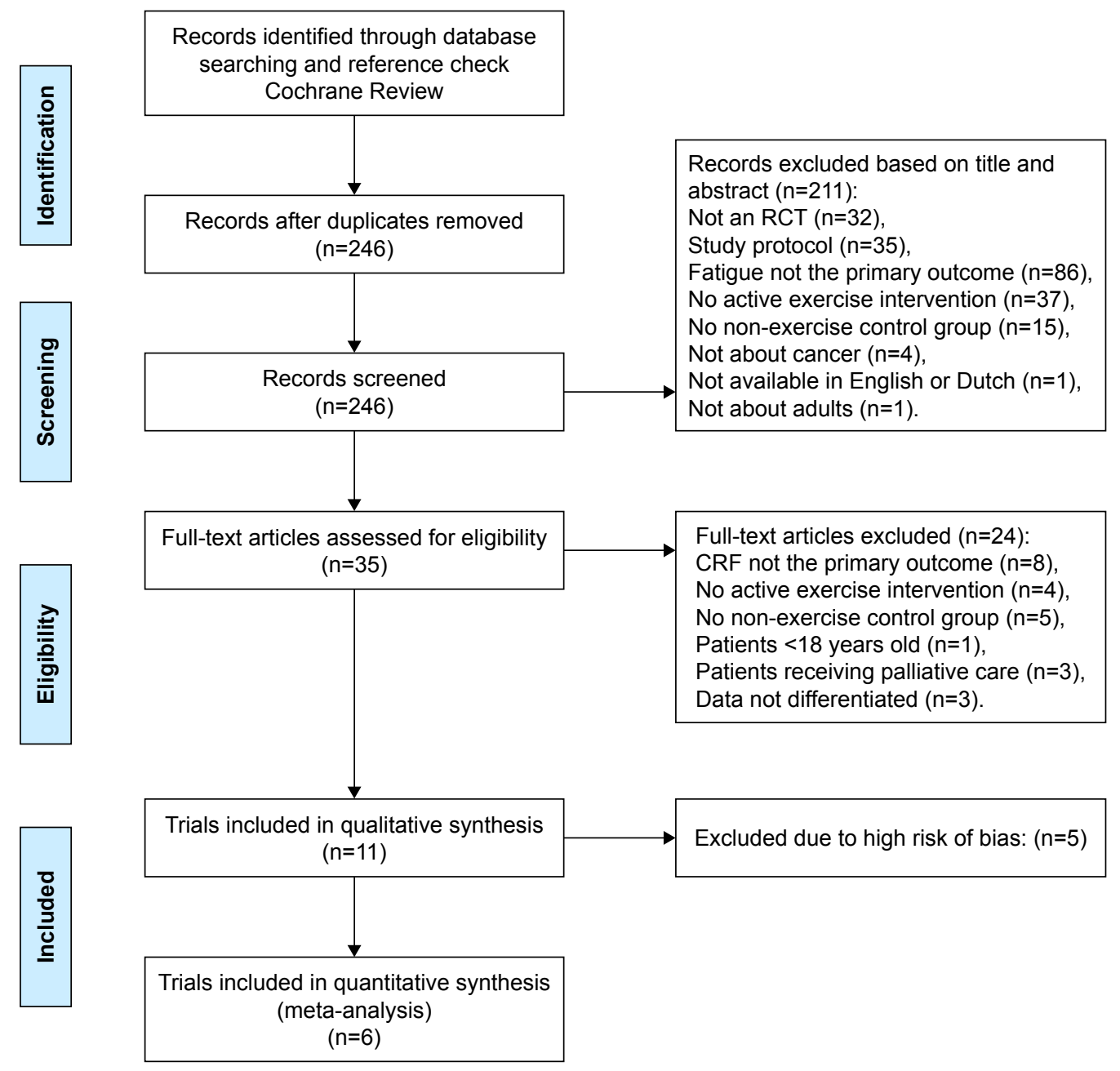

Fatigue not the primary outcome $(n=86)$,

No active exercise intervention $(n=37)$,

No non-exercise control group $(n=15)$

cancer $(n=4)$

ot available in English or Dutch $(n=1)$

Not about adults $(n=1)$.

active exercise intervention $(n=4)$

Patients $<18$ years old $(n=1)$,

Patients receiving palliative care $(n=3)$,

Data not differentiated $(n=3)$.

\section{Intervention characteristics and supervision}

Eight trials found significant improvements in $\mathrm{CRF}, 42,43,45,47-49,51$ and one found a significant effect for exercise after correcting for adherence to the intervention. ${ }^{46}$ Two found no effect on CRF. ${ }^{4450}$ Three types of exercise interventions were distinguished: aerobic exercise, ${ }^{43,44,46,49-51,52}$ resistance exercise, which was not evaluated as stand-alone exercise intervention in the selected trials; and a mix of both. ${ }^{42,45,47,48}$ Three trials offered a fully supervised exercise program at a health care location, ${ }^{42,43,48}$ eight offered home-based interventions with monitoring methods like a wristband monitor, ${ }^{50}$ follow-up calls, ${ }^{44,45}$ or logs. ${ }^{46,47,49,51,52}$ Intensity of the exercise interventions in METs varied: the exercise programs combining aerobics and resistance exercises ${ }^{42,47,48}$ and one aerobic program ${ }^{44}$

Figure I PRISMA 2009 flow diagram.

Abbreviation: CRF, cancer-related fatigue. 
were of high intensity, and the other aerobic exercise programs were of low intensity. ${ }^{43,46,49-52}$ One study did not provide enough information to determine METs: ${ }^{45}$ their patients did not keep detailed records of their home exercise.

\section{Outcome measure characteristics}

The timing of measurements varied and for the benefit of clinical relevance, and to enable us to pool similar results, we chose to use the outcomes at post-intervention, not the outcomes at follow-up, as this differed greatly between trials and long-term follow-up might depend on other factors than the intervention alone.

\section{Synthesis of results (meta-analysis)}

In order to establish the overall effect of exercise on CRF, a first meta-analysis was performed for CRF outcomes of the six low risk of bias trials. See Figure 2 for the forest plot. The effects were presented in terms of standardized effect sizes (Cohen's $d$ ). The results of the random analysis showed that any exercise improves CRF, compared to controls. The pooled estimate of the effect size was large $(0.605,95 \% \mathrm{CI}$ $0.235-0.975)$. Heterogeneity ( $Q$-value) of this effect was $Q(2)=15, p=0.010$. The $I^{2}$ statistic was $67 \%$, indicating sufficient heterogeneity to use a random model to fit the data.

\section{Pre-envisioned moderator analyses}

Several pre-envisioned moderator analyses were performed: cancer type, intervention type, MET, and adherence.

\section{Cancer type}

Two trials included patients with breast cancer, ${ }^{43,48}$ one included prostate cancer, ${ }^{47}$ one included gynecological cancers, ${ }^{45}$ and the other two trials were categorized as "mixed cancer". ${ }^{42,51}$ The moderator analysis of type of cancer showed no significant heterogeneity between these four cancer types $(Q(3)=3.7, p=0.295$ (ns)).

\section{Type of exercise intervention}

A moderator analysis of intervention type was performed. The two aerobic exercise trials ${ }^{43,51}(\Delta=1.009$, CI $0.222-$ 1.797) show a significantly greater effect than the four trials ${ }^{42,45,47,48}$ examining a combination of aerobic and resistance exercises $(\Delta=0.341$, CI $0.129-0.552)$. There was no significant heterogeneity between groups $(Q(1)=2.6$, $p=0.108$ (ns)).

\section{MET}

Using MET as a moderator to explore the role of intensity of exercise, one study ${ }^{45}$ could not be taken into account as we had insufficient data to calculate MET intensity. We performed a meta-regression analysis that showed better results for low MET intensity; however, this finding was not significant.

\section{Adherence}

The meta-regression analysis showed a significant effect of adherence on effect size $(Q(1)=5.925, p=0.01)$. With low adherence $(<56 \%)$, the effect size is 0 . With high adherence, the effect size becomes large, going up to 0.8 for $100 \%$ adherence (see Figure 3).

\section{Sensitivity analysis}

We performed an additional sensitivity analysis, including the five trials with high risk of bias. This did not change the outcome that exercise improves CRF compared to controls. The pooled effect size was diminished slightly, but still medium $(0.465,95 \%$ CI $0.217,0.712)$. Heterogeneity of this effect was $Q(10)=26, p \leq 0.01$. The $I^{2}$ statistic was $63 \%$.

\section{Publication bias}

A test for publication bias was performed. The fail-safe $N$ showed that 42 additional trials should be added to the analysis before the cumulative effect would become statistically non-significant. Given the fact that only 11 trials could be identified that specifically looked at the effect of exercise

\begin{tabular}{|c|c|c|c|c|c|c|c|c|c|}
\hline \multirow[b]{2}{*}{ Study } & \multirow[b]{2}{*}{ Outcome } & \multicolumn{5}{|c|}{ Statistics for each study } & \multirow[b]{2}{*}{ Z-value } & \multirow[b]{2}{*}{$p$-value } & \multirow[b]{2}{*}{ Std diff in means and $95 \% \mathrm{Cl}$} \\
\hline & & $\begin{array}{l}\text { Std diff } \\
\text { in means }\end{array}$ & $\begin{array}{l}\text { Standard } \\
\text { error }\end{array}$ & Variance & $\begin{array}{l}\text { Lower } \\
\text { limit }\end{array}$ & $\begin{array}{l}\text { Upper } \\
\text { limit }\end{array}$ & & & \\
\hline Adamsen et al ${ }^{41}$ & EORTC-QLQ & 0.272 & 0.131 & 0.017 & 0.015 & 0.529 & 2.076 & 0.038 & \\
\hline Cantarero-Villanueva et al ${ }^{42}$ & PFS & 1.400 & 0.271 & 0.073 & 0.869 & 1.930 & 5.173 & 0.000 & \\
\hline Donnelly et $\mathrm{al}^{44}$ & MFSI-SF & 0.207 & 0.349 & 0.122 & -0.477 & 0.892 & 0.593 & 0.553 & \\
\hline Mustian et al ${ }^{46}$ & PROMIS & 0.559 & 0.331 & 0.109 & -0.089 & 1.207 & 1.690 & 0.091 & \\
\hline Yeo et al ${ }^{50}$ & PROMIS & 0.596 & 0.302 & 0.091 & 0.003 & 1.189 & 1.971 & 0.049 & \\
\hline \multirow[t]{3}{*}{ Rogers et $\mathrm{al}^{47}$} & PROMS & 0.638 & 0.310 & 0.096 & 0.030 & 1.247 & 2.057 & 0.040 & \\
\hline & & 0.605 & 0.189 & 0.036 & 0.235 & 0.975 & 3.205 & 0.001 & \\
\hline & & & & & & & & -2.00 & -1.00 \\
\hline
\end{tabular}

Figure 2 Forest plot of effect on CRF.

Abbreviation: CRF, cancer-related fatigue; EORTC QLQ-C30, European Organisation for Research and Treatment of Cancer Quality of Life Questionnaire-Core 30; PFS, Piper Fatigue Scale; MFSI-SF, Multidimensional Fatigue Symptom Inventory-Short Form; PROMIS, Patient-Reported Outcomes Measurement Information System; Std diff, standard difference. 


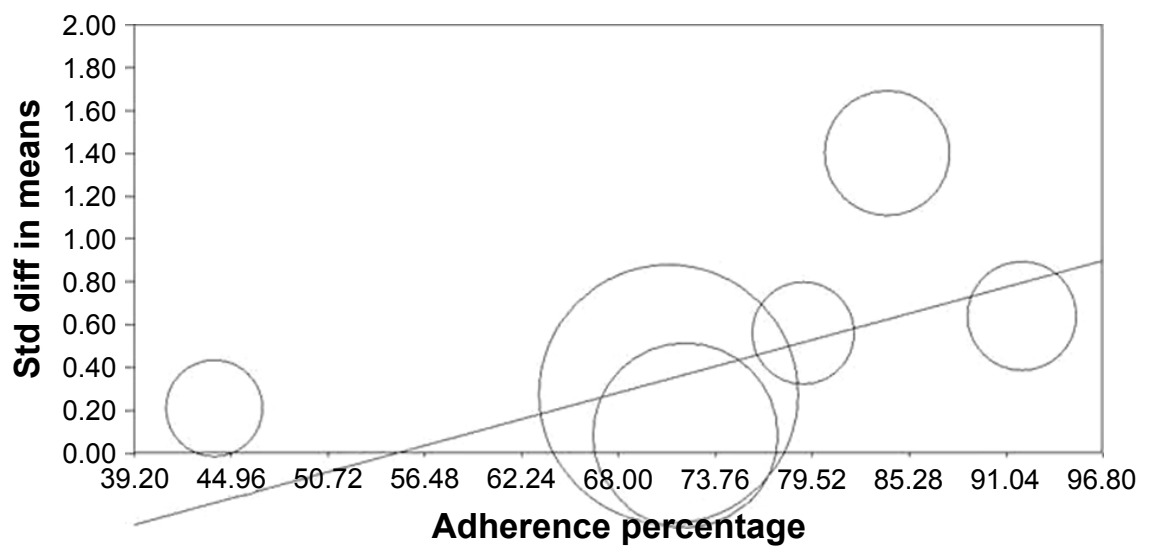

Figure 3 Meta-regression plot of adherence.

Abbreviation: Std diff in means, standard difference in means.

on $\mathrm{CRF}$ as a primary outcome compared to a non-exercise control group, and only six of those addressed risk of bias sufficiently, it is unlikely that 42 trials were missed. This indicates that no significant publication bias seems to be the case and the reported effect is valid.

\section{Discussion}

\section{Summary of evidence}

This study provides us with the possibility to estimate the effect of exercise, the surplus value of aerobic exercise, and the importance of adherence in cancer survivors who may or may not still be under treatment, but not in a terminal phase.

We found a clear improvement in CRF as a result of exercise interventions, with a large effect size. Aerobic exercises showed better improvement than a combination of aerobic and resistance exercises. Adherence to the intervention is important: high adherence resulted in a large effect, whereas low or moderate adherence yielded small effects. These effects were the same for all cancer types in the study. The indication that low-intensity exercise might be more effective than high-intensity exercise was not significant in a meta-regression.

\section{Comparison with other studies}

This finding is in sync with the National Comprehensive Cancer Network guidelines on treating CRF, ${ }^{8}$ which note that exercise is one of the most effective non-pharmacological treatments for CRF. Embedding of exercise programs in current oncologic rehabilitation guidelines is, however, still not standard. Our results clearly indicate that this should be considered.

Other studies report that CRF is highly associated with pain, insomnia, and psychological distress factors like depression, ${ }^{52}$ similar findings have been reported in CFS. ${ }^{53}$ These symptoms may arise through a common pathway, as previous research on inflammation and CRF suggests that tumours and the treatments used to treat them activate proinflammatory cytokines, leading to CRF and other symptoms. ${ }^{54}$ Similar suggestions have been made for CFS, ${ }^{55}$ as difficulty in following through with exercise has also been found in chronic fatigue patients. It has been suggested that exercise does improve fatigue; however, too much exercise might have adverse effects resulting in low adherence to the exercise protocol. ${ }^{56}$ It might be somewhat surprising that low-intensity exercise is more effective than high-intensity exercise. However, this finding is in sync with findings in CFS that overriding leads to more post-exercise malaise than pacing yourself and grading activities. ${ }^{57}$ This may be an indication that for handling fatigue, low exercise may be enough and that strenuous exercise is not needed to get good results. This finding warrants further research in treatment modes for fatigue in general.

Another important factor to address is adherence. Pathophysiological or mental barriers, or fatigue by itself could hinder patients from becoming (more) active. ${ }^{58}$ Our results show high adherence rates leading to better CRF outcomes. Information provision by health care professionals might ensure adherence; other methods are sufficient professional support, tailored advice, clear individual goals, and including the support system. ${ }^{59,60}$

\section{Implications}

The finding that exercise leads to lower levels of CRF underlines the importance of focussing upon physical training in the care of cancer patients. Exercise programs can have a direct effect on CRF by increasing muscle strength and physical fitness to counteract physical deconditioning. 
However, exercise can also have an indirect effect on CRF. CRF is highly associated with physical distress factors like pain and insomnia and psychological distress factors like depression and anxiety on CRF. Similar findings have been reported in CFS. ${ }^{54}$ It may be that these symptoms arise through a common pathway as previous research on inflammation and CRF suggests that tumours and the treatments used to treat them activate proinflammatory cytokines, leading to CRF and other symptoms. ${ }^{55}$ Similar suggestions have been made for CFS, which has as characteristic that, apart from the fatigue, other symptoms should be present, such as memory or concentration problems, muscle pain, joint pain, headache, sleeping problems, and malaise after exercise. ${ }^{56,61}$ Exercise can reduce CRF indirectly by its beneficial effects on mood, immune functioning, or sleep. ${ }^{62}$ In that sense, working mechanisms of exercise might have aspects similar to those in CFS. This should be a topic of further research.

The findings in this review may therefore be relevant not only for cancer survivors, but also, more in general, for people suffering from chronic fatigue, as it provides clinicians with concrete tools to enhance the possibility for adherence to exercise protocols. This should be a topic for future research.

\section{Limitations}

The main limitation of this study is that the number of trials was small and that only six were of low risk of bias. The randomization procedures are not addressed sufficiently to evaluate or reproduce, and allocation concealment/blinding was sometimes not mentioned at all. Moreover, in many trials, active treatment status was not addressed properly. Trials included in the review have different timeframes: some were during active treatment and others were after primary treatment or a combination of the two. There is a need for RCTs of better quality in this field. However, a sensitivity analysis including the high risk of bias trials showed that the effect of exercise versus non-exercise still remained positive.

Furthermore, most patients in our sample were "early" cancer survivors as many were still undergoing treatment during the RCT. It is unclear if our recommendations for exercise are valid for long-term cancer survivors. Research with longterm survivors and longer follow-up is clearly needed.

Furthermore, trials with widely divergent cancer types are included. Although this enables us to estimate the effect as a general intervention in all types of cancer, more trials for specific cancer types would be needed to estimate the effect in specific cancer types and their specific treatments.

\section{Strengths}

Comparison with a previous meta-analysis shows several methodological differences. We only included RCTs with non-exercise intervention controls; the ones that studied $\mathrm{CRF}$ as a primary outcome and patients receiving palliative care were not included. In contrast to the study of Cramp and Byron-Daniel, ${ }^{27}$ we were able to conduct sub-analyses for type and intensity of exercise, which enabled us to find clear cues for exercise regimens.

\section{Conclusion}

Based on this review, we conclude that exercise effectively improves CRF, especially with high adherence rates. The clinical recommendation is to improve exercise in cancer survivors with an exercise intervention that includes aerobic exercise and with high focus on facilitating adherence. Research implications are that more research of sufficient quality is needed. Future research should preferably assess CRF as a primary outcome.

\section{Acknowledgment}

Mw drs L Ossewaarde was involved in an early stage in setting up the database search. No other contributions were made.

\section{Disclosure}

A poster with preliminary results without pooled estimate was previously presented at the Dutch psychiatry association (NVvP) congress on April 10th 2014. The authors report no conflicts of interest in this work.

\section{References}

1. Cancer Research UK. Worldwide cancer incidence statistics. 2014. Available from: http://www.cancerresearchuk.org/cancer-info/cancerstats/ world/incidence. Accessed November 13, 2017.

2. US National Coalition for Cancer Survivorship. Defining cancer survivorship. Available from: http://www.canceradvocacy.org/news/definingcancer-survivorship. Accessed November 13, 2017.

3. Akechi T, Kugaya A, Okamura H, Yamawaki S, Uchitomi Y. Fatigue and its associated factors in ambulatory cancer patients: a preliminary study. J Pain Symptom Manage. 1999;17(1):42-48.

4. Hofman M, Morrow GR, Roscoe JA, et al. Cancer patients' expectations of experiencing treatment-related side effects: a University of Rochester Cancer Center-Community Clinical Oncology Program study of 938 patients from community practices. Cancer. 2004;101(4):851-857.

5. Patrick DL, Ferketich SL, Frame PS, et al; National Institutes of Health State-of-the-Science Panel. National Institutes of Health State-of-theScience Conference Statement: symptom management in cancer: pain, depression, and fatigue, July 15-17, 2002. J Natl Cancer Inst Monogr. 2004;(32):9-16.

6. Cella D, Davis K, Breitbart W, Curt G; Fatigue Coalition. Cancer-related fatigue: prevalence of proposed diagnostic criteria in a United States sample of cancer survivors. J Clin Oncol. 2001;19(14):3385-3391.

7. Wang XS, Zhao F, Fisch MJ, et al. Prevalence and characteristics of moderate to severe fatigue: a multicenter study in cancer patients and survivors. Cancer. 2014;120(3):425-432. 
8. NCCN Clinical Practice Guidelines in Oncology. Cancer-related fatigue. Version 2. 2017. Available from: https://www.nccn.org/professionals/ physician_gls/pdf/fatigue.pdf. Accessed November 13, 2017.

9. Wu HS, McSweeney M. Cancer-related fatigue: "It's so much more than just being tired". Eur J Oncol Nurs. 2007;11(2):117-125.

10. Son CG. Review of the prevalence of chronic fatigue worldwide. J Korean Orient Med. 2012;33(2):25-33.

11. Mustian KM, Alfano CM, Heckler C, et al. Comparison of pharmaceutical, psychological, and exercise treatments for cancer-related fatigue: a meta-analysis. JAMA Oncol. 2017;3(7):961-968.

12. Hall AE, Boyes AW, Bowman J, Walsh RA, James EL, Girgis A. Young adult cancer survivors' psychosocial well-being: a cross-sectional study assessing quality of life, unmet needs, and health behaviors. Support Care Cancer. 2012;20(6):1333-1341.

13. Wessely S, Powell R. Fatigue syndromes: a comparison of chronic "postviral" fatigue with neuromuscular and affective disorders. J Neurol Neurosurg Psychiatry. 1989;52(8):940-948.

14. Sharpe M, Goldsmith KA, Johnson AL, Chalder T, Walker J, White PD. Rehabilitative treatments for chronic fatigue syndrome: long-term followup from the PACE trial. Lancet Psychiatry. 2015;2(12):1067-1074.

15. Larun L, Brurberg KG, Odgaard-Jensen J, Price JR. Exercise therapy for chronic fatigue syndrome. Cochrane Database Syst Rev. 2015;(2): CD003200.

16. Kellner R, Sheffield BF. The one-week prevalence of symptoms in neurotic patients and normals. Am J Psychiatry. 1973;50:734-741.

17. Humpel N, Iverson DC. Depression and quality of life in cancer survivors: is there a relationship with physical activity? Int J Behav Nutr Phys Act. 2007;4:65.

18. Midtgaard J, Baadsgaard MT, Møller T, et al. Self-reported physical activity behaviour; exercise motivation and information among Danish adult cancer patients undergoing chemotherapy. Eur J Oncol Nurs. 2009; 13(2):116-121.

19. Drewnowski A, Evans WJ. Nutrition, physical activity, and quality of life in older adults: summary. J Gerontol A Biol Sci Med Sci. 2001; 56 Spec No 2:89-94.

20. Jones LW, Courneya KS, Vallance JK, et al. Understanding the determinants of exercise intentions in multiple myeloma cancer survivors: an application of the theory of planned behavior. Cancer Nurs. 2006;29(3):167-175.

21. Servaes P, van der Werf S, Prins J, Verhagen S, Bleijenberg G. Fatigue in disease-free cancer patients compared with fatigue in patients with chronic fatigue syndrome. Support Care Cancer. 2001;9(1):11-17.

22. Williams TE, Chalder T, Sharpe M, White PD. Heterogeneity in chronic fatigue syndrome - empirically defined subgroups from the PACE trial. Psychol Med. 2017;47(8):1454-1465.

23. Van Houdenhove B, Luyten P. Chronic fatigue syndrome reflects loss of adaptability. J Intern Med. 2010;268(3):249-251.

24. Mendoza TR, Wang XS, Cleeland CS, et al. The rapid assessment of fatigue severity in cancer patients: use of the Brief Fatigue Inventory. Cancer. 1999;85(5):1186-1196.

25. Servaes P, Gielissen MF, Verhagen S, Bleijenberg G. The course of severe fatigue in disease-free breast cancer patients: a longitudinal study. Psychooncology. 2007;16(9):787-795.

26. Pachman DR, Barton DL, Swetz KM, Loprinzi CL. Troublesome symptoms in cancer survivors: fatigue, insomnia, neuropathy, and pain. J Clin Oncol. 2012;30(30):3687-3696.

27. Cramp F, Byron-Daniel J. Exercise for the management of cancerrelated fatigue in adults. Cochrane Database Syst Rev. 2012;11: CD006145.

28. Kessels E, Husson O, van der Feltz-Cornelis CM. Review protocol. 2017. Available from: http://www.crd.york.ac.uk/PROSPERO/ display_record.asp?ID=CRD42013003670. Accessed January 10, 2018.

29. Moher D, Liberati A, Tetzlaff J, Altman DG; PRISMA Group. Preferred reporting items for systematic reviews and meta-analyses: the PRISMA statement. J Clin Epidemiol. 2009;62(10):1006-1012.

30. Larson-Meyer DE. A systematic review of the energy cost and metabolic intensity of yoga. Med Sci Sports Exerc. 2016;48(8):1558-1569.
31. Ainsworth BE, Haskell WL, Whitt MC, et al. Compendium of physical activities: an update of activity codes and MET intensities. Med Sci Sports Exerc. 2000;32(9 Suppl):S498-S504.

32. Higgins JP, Altman DG, Gøtzsche PC, et al; Cochrane Bias Methods Group; Cochrane Statistical Methods Group. The Cochrane Collaboration's tool for assessing risk of bias in randomised trials. $B M J$. 2011;343:d5928.

33. Cohen J. Statistical Power Analysis for the Behavioral Sciences. 2nd ed. Hillsdale, NJ: Lawrence Erlbaum Associates; 1988.

34. Higgins JP, Thompson SG, Deeks JJ, Altman DG. Measuring inconsistency in meta-analyses. BMJ. 2003;327(7414):557-560.

35. Borenstein M, Hedges LV, Higgins JPT, Rothstein HR. Comprehensive Meta-analysis. (Version 2.2.027) [Computer software]. Englewood, NJ: Biostat; 2005.

36. Begg CB. Publication bias. In: Cooper H, Hedges LV, editors. The Handbook of Research Synthesis. New York: Russell Sage Foundation; 1994:399-409.

37. Borenstein M, Hedges LV, Higgins JPT, Rothstein HR. Introduction to Meta-analysis (Statistics in Practice). Chichester, UK: John Wiley \& Sons; 2009.

38. Sharon A Plowman, Denise L Smith. Exercise Physiology for Health, Fitness, and Performance. Philadelphia, Lippincott Williams \& Wilkins; 2001:61.

39. William D McArdle, Frank I Katch, Victor L Katch. Essentials of Exercise Physiology. Philadelphia, Lippincott Williams \& Wilkins; 2006:204.

40. Peterson MD, Gordon PM. "Resistance exercise for the aging adult: clinical implications and prescription guidelines". Am J Med. 2011; 124(3):194-198.

41. Adamsen L, Quist M, Andersen C, et al. Effect of a multimodal high intensity exercise intervention in cancer patients undergoing chemotherapy: randomised controlled trial. BMJ. 2009;339: b3410.

42. Cantarero-Villanueva I, Fernández-Lao C, Cuesta-Vargas AI, Del Moral-Avila R, Fernández-de-Las-Peñas C, Arroyo-Morales M. The effectiveness of a deep water aquatic exercise program in cancer-related fatigue in breast cancer survivors: a randomized controlled trial. Arch Phys Med Rehabil. 2013;94(2):221-230.

43. Dodd MJ, Cho MH, Miaskowski C, et al. A randomized controlled trial of home-based exercise for cancer-related fatigue in women during and after chemotherapy with or without radiation therapy. Cancer Nurs. 2010;33(4):245-257.

44. Donnelly CM, Blaney JM, Lowe-Strong A, et al. A randomised controlled trial testing the feasibility and efficacy of a physical activity behavioural change intervention in managing fatigue with gynaecological cancer survivors. Gynecol Oncol. 2011;122(3):618-624.

45. Mock V, Frangakis C, Davidson NE, et al. Exercise manages fatigue during breast cancer treatment: a randomized controlled trial. Psychooncology. 2005;14(6):464-477.

46. Mustian KM, Peppone L, Darling TV, Palesh O, Heckler CE, Morrow GR. A 4-week home-based aerobic and resistance exercise program during radiation therapy: a pilot randomized clinical trial. J Support Oncol. 2009;7(5):158-167.

47. Rogers LQ, Vicari S, Trammell R, et al. Biobehavioral factors mediate exercise effects on fatigue in breast cancer survivors. Med Sci Sports Exerc. 2014;46(6):1077-1088.

48. Wang YJ, Boehmke M, Wu YW, Dickerson SS, Fisher N. Effects of a 6-week walking program on Taiwanese women newly diagnosed with early-stage breast cancer. Cancer Nurs. 2011;34(2):E1-E13.

49. Windsor PM, Nicol KF, Potter J. A randomized, controlled trial of aerobic exercise for treatment-related fatigue in men receiving radical external beam radiotherapy for localized prostate carcinoma. Cancer. 2004;101(3):550-557.

50. Yeo TP, Burrell SA, Sauter PK, et al. A progressive postresection walking program significantly improves fatigue and health-related quality of life in pancreas and periampullary cancer patients. J Am Coll Surg. 2012;214(4):463-475. discussion 475-477. 
51. Yuen HK, Sword D. Home-based exercise to alleviate fatigue and improve functional capacity among breast cancer survivors. J Allied Health. 2007;36(4):e257-e275.

52. Seo Y, Oh H, Seo W. Causal relationships among factors associated with cancer-related fatigue. Eur J Oncol Nurs. 2010;14(5):380-386.

53. Kempke S, Goossens L, Luyten P, Bekaert P, Van Houdenhove B, Van Wambeke P. Predictors of outcome in a multi-component treatment program for chronic fatigue syndrome. J Affect Disord. 2010;126(1-2): 174-179.

54. Bower JE, Lamkin DM. Inflammation and cancer-related fatigue: mechanisms, contributing factors, and treatment implications. Brain Behav Immun. 2013;30 Suppl:S48-S57.

55. Van Houdenhove B, Van Den Eede F, Luyten P. Does hypothalamicpituitary-adrenal axis hypofunction in chronic fatigue syndrome reflect a 'crash' in the stress system? Med Hypotheses. 2009;72(6):701-705.

56. Williams TE, Chalder T, Sharpe M, White PD. Heterogeneity in chronic fatigue syndrome - empirically defined subgroups from the PACE trial. Psychol Med. 2017:1-12.

57. Van Houdenhove B, Bruyninckx K, Luyten P. In search of new balance. Can high "action-proneness" in patients with chronic fatigue syndrome be changed by a multidisciplinary group treatment? J Psychosom Res. 2006;60(6):623-625.
58. Courneya KS, Friedenreich CM, Quinney HA, et al. A longitudinal study of exercise barriers in colorectal cancer survivors participating in a randomized controlled trial. Ann Behav Med. 2005;29(2): $147-153$.

59. Husebø AM, Karlsen B, Allan H, Søreide JA, Bru E. Factors perceived to influence exercise adherence in women with breast cancer participating in an exercise programme during adjuvant chemotherapy: a focus group study. J Clin Nurs. 2015;24(3-4):500-510.

60. Piotrowicz E, Orzechowski P, Chrapowicka A, Piotrowicz R. How should we advise heart failure patients on exercise and what should we tell them? Curr Heart Fail Rep. 2014;11(3):274-280.

61. van Houdenhove B, Heijnen CJ. [Chronic fatigue syndrome: a psychoneuroimmunological perspective]. Tijdschr Psychiatr. 2009;51(8): 603-610. Dutch.

62. Fukuda K, Straus SE, Hickie I, Sharpe MC, Dobbins JG, Komarfoff A. The chronic fatigue syndrome: a comprehensive approach to its definition and study. International Chronic Fatigue Syndrome Study Group. Ann Intern Med. 1994;121(12):953-959. 


\section{Supplementary material}

\begin{tabular}{|c|c|c|c|}
\hline Section/topic & \# & Checklist item & $\begin{array}{l}\text { Reported } \\
\text { on page } \\
\text { \# }\end{array}$ \\
\hline \multicolumn{4}{|l|}{ TITLE } \\
\hline Title & I & Identify the report as a systematic review, meta-analysis, or both. & I \\
\hline \multicolumn{4}{|l|}{ ABSTRACT } \\
\hline $\begin{array}{l}\text { Structured } \\
\text { summary }\end{array}$ & 2 & $\begin{array}{l}\text { Provide a structured summary including, as applicable, background; objectives; data sources; study } \\
\text { eligibility criteria, participants, and interventions; study appraisal and synthesis methods; results; } \\
\text { limitations; conclusion and implications of key findings; systematic review registration number. }\end{array}$ & 3 \\
\hline \multicolumn{4}{|c|}{ INTRODUCTION } \\
\hline Rationale & 3 & Describe the rationale for the review in the context of what is already known. & 5 \\
\hline Objectives & 4 & $\begin{array}{l}\text { Provide an explicit statement of questions being addressed with reference to participants, interventions, } \\
\text { comparisons, outcomes, and study design (PICOS). }\end{array}$ & 6 \\
\hline \multicolumn{4}{|l|}{ METHODS } \\
\hline $\begin{array}{l}\text { Protocol and } \\
\text { registration }\end{array}$ & 5 & $\begin{array}{l}\text { Indicate if a review protocol exists, if and where it can be accessed (eg, web address), and, if available, } \\
\text { provide registration information including registration number. }\end{array}$ & 6 \\
\hline $\begin{array}{l}\text { Eligibility } \\
\text { criteria }\end{array}$ & 6 & $\begin{array}{l}\text { Specify study characteristics (eg, PICOS, length of follow-up) and report characteristics (eg, years } \\
\text { considered, language, publication status) used as criteria for eligibility, giving rationale. }\end{array}$ & 6 \\
\hline $\begin{array}{l}\text { Information } \\
\text { sources }\end{array}$ & 7 & $\begin{array}{l}\text { Describe all information sources (eg, databases with dates of coverage, contact with study authors to } \\
\text { identify additional studies) in the search and date last searched. }\end{array}$ & 7 \\
\hline Search & 8 & $\begin{array}{l}\text { Present full electronic search strategy for at least one database, including any limits used, such that it } \\
\text { could be repeated. }\end{array}$ & 7 \\
\hline Study selection & 9 & $\begin{array}{l}\text { State the process for selecting studies (ie, screening, eligibility, included in systematic review, and, if } \\
\text { applicable, included in the meta-analysis). }\end{array}$ & 7 \\
\hline $\begin{array}{l}\text { Data collection } \\
\text { process }\end{array}$ & 10 & $\begin{array}{l}\text { Describe method of data extraction from reports (eg, piloted forms, independently, in duplicate) and any } \\
\text { processes for obtaining and confirming data from investigators. }\end{array}$ & 8 \\
\hline Data items & 11 & $\begin{array}{l}\text { List and define all variables for which data were sought (eg, PICOS, funding sources) and any assumptions } \\
\text { and simplifications made. }\end{array}$ & 8 \\
\hline $\begin{array}{l}\text { Risk of bias in } \\
\text { individual trials }\end{array}$ & 12 & $\begin{array}{l}\text { Describe methods used for assessing risk of bias of individual studies (including specification of whether } \\
\text { this was done at the study or outcome level), and how this information is to be used in any data synthesis. }\end{array}$ & 8 \\
\hline $\begin{array}{l}\text { Summary } \\
\text { measures }\end{array}$ & 13 & State the principal summary measures (eg, risk ratio, difference in means). & 9 \\
\hline $\begin{array}{l}\text { Synthesis of } \\
\text { results }\end{array}$ & 14 & $\begin{array}{l}\text { Describe the methods of handling data and combining results of studies, if done, including measures of } \\
\text { consistency }\left(\mathrm{eg}, \mathbb{R}^{2}\right) \text { for each meta-analysis. }\end{array}$ & 9 \\
\hline $\begin{array}{l}\text { Risk of bias } \\
\text { across trials }\end{array}$ & 15 & $\begin{array}{l}\text { Specify any assessment of risk of bias that may affect the cumulative evidence (eg, publication bias, } \\
\text { selective reporting within studies). }\end{array}$ & 9 \\
\hline $\begin{array}{l}\text { Additional } \\
\text { analyses }\end{array}$ & 16 & $\begin{array}{l}\text { Describe methods of additional analyses (eg, sensitivity or subgroup analyses, meta-regression), if done, } \\
\text { indicating which were pre-specified. }\end{array}$ & 9 \\
\hline \multicolumn{4}{|l|}{ RESULTS } \\
\hline Study selection & 17 & $\begin{array}{l}\text { Give numbers of studies screened, assessed for eligibility, and included in the review, with reasons for } \\
\text { exclusions at each stage, ideally with a flow diagram. }\end{array}$ & 10 \\
\hline $\begin{array}{l}\text { Study } \\
\text { characteristics }\end{array}$ & 18 & $\begin{array}{l}\text { For each study, present characteristics for which data were extracted (eg, study size, PICOS, follow-up } \\
\text { period) and provide the citations. }\end{array}$ & II \\
\hline $\begin{array}{l}\text { Risk of bias } \\
\text { within studies }\end{array}$ & 19 & Present data on risk of bias of each study and, if available, any outcome level assessment (see item 12). & II \\
\hline $\begin{array}{l}\text { Results of } \\
\text { individual trials }\end{array}$ & 20 & $\begin{array}{l}\text { For all outcomes considered (benefits or harms), present, for each study: (a) simple summary data for } \\
\text { each intervention group, (b) effect estimates and confidence intervals, ideally with a forest plot. }\end{array}$ & II \\
\hline $\begin{array}{l}\text { Synthesis of } \\
\text { results }\end{array}$ & 21 & Present results of each meta-analysis done, including confidence intervals and measures of consistency. & 12 \\
\hline $\begin{array}{l}\text { Risk of bias } \\
\text { across studies }\end{array}$ & 22 & Present results of any assessment of risk of bias across studies (see item I5). & 14 \\
\hline $\begin{array}{l}\text { Additional } \\
\text { analysis }\end{array}$ & 23 & $\begin{array}{l}\text { Give results of additional analyses, if done (eg, sensitivity or subgroup analyses, meta-regression } \\
\text { [see item 16]). }\end{array}$ & 13 \\
\hline
\end{tabular}

Figure SI (Continued) 


\begin{tabular}{|c|c|c|c|}
\hline Section/topic & $\#$ & Checklist item & $\begin{array}{l}\text { Reported } \\
\text { on page } \\
\#\end{array}$ \\
\hline \multicolumn{4}{|l|}{ DISCUSSION } \\
\hline $\begin{array}{l}\text { Summary of } \\
\text { evidence }\end{array}$ & 24 & $\begin{array}{l}\text { Summarize the main findings including the strength of evidence for each main outcome; consider their } \\
\text { relevance to key groups (eg, health care providers, users, and policy makers). }\end{array}$ & 14 \\
\hline Limitations & 25 & $\begin{array}{l}\text { Discuss limitations at study and outcome level (eg, risk of bias), and at review-level (eg, incomplete } \\
\text { retrieval of identified research, reporting bias). }\end{array}$ & 16 \\
\hline Conclusion & 26 & $\begin{array}{l}\text { Provide a general interpretation of the results in the context of other evidence, and implications for } \\
\text { future research. }\end{array}$ & 16 \\
\hline \multicolumn{4}{|l|}{ FUNDING } \\
\hline Funding & 27 & $\begin{array}{l}\text { Describe sources of funding for the systematic review and other support (eg, supply of data); role of } \\
\text { funders for the systematic review. }\end{array}$ & 2 \\
\hline
\end{tabular}

Figure SI PRISMA checklist.

\section{Publish your work in this journal}

Neuropsychiatric Disease and Treatment is an international, peerreviewed journal of clinical therapeutics and pharmacology focusing on concise rapid reporting of clinical or pre-clinical studies on a range of neuropsychiatric and neurological disorders. This journal is indexed on PubMed Central, the 'PsycINFO' database and CAS, and is the official journal of The International Neuropsychiatric Association (INA). The manuscript management system is completely online and includes a very quick and fair peer-review system, which is all easy to use. Visit http://www.dovepress.com/testimonials.php to read real quotes from published authors. 